Trauma Cranioencefálico na Infância

Bartira de Godoy Maranhão Santos - Flávia Cristina Malheiros Hayashi - Adriana Vaz de Oliveira e Silva - Cinthia Ferreira da Silva - Rodrigo Eustáquio

Reabilitação e Neurologia

\title{
Trauma Cranioencefálico na Infância
}

\author{
Bartira de Godoy Maranhão Santos, Flávia Cristina Malheiros Hayashi, Adriana \\ Vaz de Oliveira e Silva, Cinthia Ferreira da Silva, Rodrigo Eustáquio
}

Acadêmicos da Universidade Severino Sombra na Disciplina Neurologia

Ana Beatriz Calmon N. da G. Pereira

Professora da Universidade Severino Sombra na Disciplina Neurologia, anacalmon@uol.com.br

Resumo: o trauma crânio-encefálico (TCE) grave é uma das mais prevalentes causas de morte e de deficiência física. É considerada uma das patologias de maior impacto na qualidade de vida. Mas os principais tipos de lesões são, no parênquima cerebra, cerca de 55\% dos casos; contusões cerebrais, $45 \%$ a 60\% das lesões traumáticas primárias, além de outras lesões intracranianas como o hematoma subdural agudo e hemorragia meníngea traumática. As alterações neuropsicológicas, que podem também aparecer, são numerosas e diversificadas e dependem de fatores como a gravidade do traumatismo, características do dano cerebral e das zonas afetadas (tamanho e localização) e características pré-mórbidas do sujeito. Descrevemos o caso de uma criança de 11 anos, do sexo masculino, residente em Vassouras-RJ, vítima de TCE em atividade de recreação. Na tomografia computadorizada foi evidenciada uma lesão temporo-parietal, com exposição de massa encefálica; presença de focos hemorrágicos; edema difuso e áreas de necrose cerebral. Mas que cursaram com sequelas significativas.

Palavras-Chave: Trauma cranioencefálico. Criança. Sequelas.

\section{Cranioencephalus Trauma in Childhood}

\begin{abstract}
CET) is one of the prevalent dead causes and of physical deficiency would be considered one of the diseases of major impact on quality of life. The main types of lesions are: in cerebral parenchyma in about of $55 \%$ of the cases, cerebral concussions in $45 \%$ to $60 \%$ of the lesions traumatic primary, beside of others intracranial lesions as the acute subdural hematoma and traumatic meninges hemorrhagic. The neuropsychological alterations that also could appear are numerous and different and depend of factors as the traumatism, cerebral injury characteristics and the affected zones (localization and size) and subject post-
\end{abstract}

Rev. de Saúde, Vassouras, v. 1, n. 1, p. 07-14, jan./mar., 2010 
Trauma Cranioencefálico na Infância

Bartira de Godoy Maranhão Santos - Flávia Cristina Malheiros Hayashi - Adriana Vaz de Oliveira e Silva - Cinthia Ferreira da Silva - Rodrigo Eustáquio

Reabilitação e Neurologia

morbid characteristics. We will describe the case of a child of 11 years old, male, of Vassouras-RJ, presented CET in recreation activity. In computed tomography was evidenced a temporo-parietal lesion with exposition of encephalic mass, presence of hemorrhagic regions, diffuse edema, cerebral necrosis areas, and that cursed with significant damage.

Keywords: Cranioenfephalus trauma. Child. Injury.

\section{Introdução}

O Traumatismo Crânioencefálico (TCE) constitui um dos maiores problemas de saúde pública, é uma das mais prevalentes causas de mortalidade em países industrializados e uma das principais causas de morte e de sequelas em crianças e adolescentes no mundo. O Trauma Cranioencefálico está presente na maioria das crianças vítimas de traumatismo e é responsável por mais de $75 \%$ das mortes na infância. Para cada paciente morto, três ficam com graves sequelas [Carvalho et al. 2007]. Crianças com TCE grave necessitam de internação prolongada em Unidade de Terapia Intensiva (UTI), com alta mortalidade e morbidade, além de elevado custo financeiro. Entre as crianças que não morrem, existe uma percentagem significativa que apresenta dificuldades comportamentais e de aprendizagem, e estima-se que $80 \%$ com traumatismo grave, têm dificuldades educacionais especiais ou necessitam de modificações no ambiente educacional, dois anos após o acidente [Carvalho et al. 2007]; [Lobato 2006].

Conceitua-se como TCE qualquer agressão que acarrete lesão anatômica ou comprometimento funcional do couro cabeludo, crânio, meninges ou encéfalo, causada por uma força física externa [Nitrini e Bacheschi 2005], com sequelas significativas tanto no âmbito motor quanto no psicológico. É classificado quanto ao mecanismo, como gravidade ou morfologia. Em relação ao mecanismo a lesão pode ser fechada (contusa) ou penetrante. As lesões fechadas, mais comuns na infância, são causadas por quedas, atropelamentos, acidentes automobilísticos e agressões. A gravidade é baseada na escala de coma de Glasgow (ECG), podendo ser leve (ECG de 14 a 15), moderada (ECG de 9 a 13) ou grave (ECG de 3 a 8). Esta escala é utilizada também como parâmetro evolutivo e índice prognóstico. Morfologicamente dividi-se em Lesões Extracranianas (lacerações de couro cabeludo que podem ser fonte importante de sangramento e hematomas subgaleais); Fraturas de Crânio (lineares, cominutivas, com afundamento, geralmente associadas com lesão de dura-máter e do parênquima cerebral); e Lesões intracranianas que podem ser focais (hematomas extradural, subdural ou intra-parenquimatoso) ou difusas (concussão, lesão axonal difusa ou edema e ingurgitamento cerebral) [Carvalho et al. 2007].

O conhecimento das causas do Traumatismo Cranioencefálico Grave em crianças e adolescentes possibilita a implantação de medidas para prevenção primária, que visam à redução do número de traumas, ao passo que, a determinação dos fatores relacionados com o óbito, propicia a elaboração de protocolos de tratamento para reduzir a morbidade e a mortalidade, denominada prevenção secundária. A experiência clínica e os trabalhos científicos têm mostrado um prognóstico mais favorável nas crianças com TCE que nos adultos.Quase sempre as taxa de mortalidade do TCE grave em Pediatria, variam entre

Rev. de Saúde, Vassouras, v. 1, n. 1, p. 07-14, jan./mar., 2010 
Trauma Cranioencefálico na Infância

Bartira de Godoy Maranhão Santos - Flávia Cristina Malheiros Hayashi - Adriana Vaz de Oliveira e Silva - Cinthia Ferreira da Silva - Rodrigo Eustáquio

Reabilitação e Neurologia

trabalhos publicados, de 10 a $50 \%$. Nesses casos, uma boa recuperação muitas vezes, não significa recuperação total, e muitas crianças que sofrem de TCE apresentam dificuldades temporárias ou permanentes quanto à cognição, da memória ou deficiência física.

\section{Metodologia}

O nosso estudo teve como foco relatar e avaliar o caso de um paciente de 11 anos, que sofreu um TCE em 22/04/2008, comparando-o com a literatura. O levantamento literário foi feito através de consultas a artigos científicos obtidos na biblioteca da Universidade Severino Sombra (USS) e em sites de pesquisa da internet, além de livros, todos citados na referência bibliográfica.

A responsável pelo paciente, após ser esclarecida sobre o trabalho, assinou o termo de consentimento livre e esclarecido e transmitiu o acesso ao prontuário, ao uso de seus exames laboratoriais e de imagem para publicação.

A anamnese e o exame físico foram feitos de forma dirigida para possíveis patologias neurológicas relacionadas com o trauma sofrido pela criança, e estes foram complementados com o miniexame do estado mental, que avalia as funções cognitivas especificas como orientação temporal e espacial; memória; atenção e cálculo; linguagem e capacidade construtiva visual.

\section{Relato de Caso}

Descrevemos um paciente pré-adolescente, L.T.M., 11 anos, do gênero masculino, residente em Vassouras que foi encaminhado ao serviço de emergência no dia 22 de abril de 2008, após sofrer Traumatismo Crânio Encefálico (TCE) grave em atividade recreativa.

Paciente deu entrada no Hospital Universitário Sul Fluminense (HUSF) da Universidade Severino Sombra, com TCE grave, hemorragia subaracnoidea e exposição de massa encefálica. Apresentou score 7 na escala de coma de Glasgow (sem resposta ocular - 1; localizando a dor -5 ; sem resposta verbal - 1), sendo imediatamente entubado.

Ao exame físico, observou-se paciente hipocorado $++/ 4+$, anisocórico (pupila esquerda maior que a direita), pupila esquerda não fotorreagente, hipertropia de olho esquerdo, sangramento e lesão cortocontusa em região parietal esquerda, com exposição de massa encefálica, reflexo de tronco positivo, reação aos estímulos álgicos, pressão arterial em membro superior direito de 100x60 mmHg, frequência cardíaca de $108 \mathrm{bpm}$, RCR 2T, com bulhas normofonéticas, frequência respiratória de 15 irpm; abdome flácido sem massas e/ou vísceromegalias, A gasometria apresentava os seguintes resultados: $\mathrm{pH}$ : 7,233, $\mathrm{pO}_{2} 147,3 \mathrm{mmHg}, \mathrm{pCO}_{2} 31,6 \mathrm{mmHg}, \mathrm{HCO}_{3} 15,3 \mathrm{mmol} / \mathrm{L}, \mathrm{TCO}_{2} 16,2 \mathrm{mmol} / \mathrm{L}$.

Submetido à tomografia computadorizada (TC) de crânio (22/04/08), foi evidenciada fratura têmporo-parietal esquerda, edema difuso importante e hemorragia subaracnóide sendo encaminhado ao Centro de Terapia Intensiva. Realizou-se, tratamento 
Trauma Cranioencefálico na Infância

Bartira de Godoy Maranhão Santos - Flávia Cristina Malheiros Hayashi - Adriana Vaz de Oliveira e Silva - Cinthia Ferreira da Silva - Rodrigo Eustáquio

Reabilitação e Neurologia

conservador do TCE grave, o paciente foi sedado com Midazolam Rawsay $\mathrm{V}(0,2 \mathrm{mg} / \mathrm{kg} / \mathrm{h})$ e permaneceu em ventilação mecânica, hidratação vigorosa, manitol, fenitoína. Encaminhado à Neurocirurgia onde foi feito reparo por lavagem e sutura da lesão e do couro cabeludo e curativo, manteve-se a medicação antes prescrita e iniciouse antibioticoterapia profilática (ceftriaxone e oxacilina). Uma nova TC foi solicitada em 48 horas, e realizada no dia 25/04/08, cujas as mesmas alterações foram visualizadas.

Paciente permaneceu durante quatorze dias em ventilação mecânica, adquiriu pneumonia por Acinetobacter e foi medicado com Imipenem, Tetraciclina e Meropenem o que se resolveu em 15 dias.

Na TC de crânio (06/05/08) manteve edema cerebral com focos hemorrágicos e higroma. Ao exame físico apresentou aparelho locomotor com musculatura globalmente hipotrófica , diminuição de força importante em hemisfério direito, devido à sequela neurológica, ausência de motricidade voluntária fina respondendo somente a estímulos verbais e visuais, através da cabeça e membro esquerdo além de disfagia. Paciente fazia fonoaudiologia e fisioterapia diariamente e aguardava alta após desmame da sonda nasogástrica. No dia 11/06/08, foi submetido a outra TC, que evidenciava lesão temporo-parietal, presença de focos hemorrágicos, discreta atrofia cerebral e áreas de necrose cerebral.

$\mathrm{Na}$ última avaliação, $51^{\circ}$ dia de avaliação na Enfermaria, L.T.M. apresentava-se com pneumonia tratada, sem sonda nasogástrica e submetido à dieta oral. Ao exame físico: bom estado geral, corado, hidratado, acianótico, anictérico, afebril, FC 80-96 bpm, FR 14-22 irpm, PA 120/80mmHg, RCV em 2T, com bulhas normofonéticas sem sopro sistólico, murmúrio vesicular audível sem ruídos adventícios, abdome flácido, peristáltico, indolor, hábitos intestinais normais, diurese presente, pouco colaborativo no exame e no tratamento fisioterapêutico recebeu alta hospitalar.

Atualmente, o paciente apresenta à ectoscopia bom estado geral, normocorado, anictérico, acianótico e ao exame neurológico encontra-se consciente, desorientado alopsiquicamente. $\mathrm{Na}$ avaliação da linguagem encontramos: distúrbio da comunicação verbal (afasia de Broca) e distúrbio da nomeação (agnosia tátil e visual).

No exame dos nervos cranianos o paciente tem alteração dos nervos cranianos III, IV e VI e demonstra incapacidade da musculatura extrínseca em realizar abdução, adução e abaixamento do globo ocular. Foram observadas alterações no nervo VII, constatou-se uma paralisia facial periférica, com redução significativa da mímica facial. $\mathrm{Na}$ avaliação do XII, par craniano, observou-se discreta redução da motilidade do lado direito da língua.

$\mathrm{Na}$ avaliação da sensibilidade o paciente apresentou hipoestesia superficial (táctil e dolorosa) de $++++/ 5+$ nos membros superior e inferior do lado direito, já que a sensibilidade térmica e a proprioceptiva estavam conservadas. No exame do aparelho locomotor, os testes de força muscular mostraram uma paresia facio-braquio-cural grau 3/5, que pode ser constatada através da Manobra de Mingazzini. Na inspeção dos segmentos corporais tem discreta hipotrofia em hemicorpo direito. Na pesquisa do tônus muscular e dos reflexos apresenta hipertonia espástica e hiperreflexia profunda 
Trauma Cranioencefálico na Infância

Bartira de Godoy Maranhão Santos - Flávia Cristina Malheiros Hayashi - Adriana Vaz de Oliveira e Silva - Cinthia Ferreira da Silva - Rodrigo Eustáquio

Reabilitação e Neurologia

(principalmente reflexo patelar), Sinal de Babinski (reflexo cutaneoplantar em extensão).

Em relação à coordenação motora do paciente pudemos comprovar alterações através das provas índex-nariz, supinação-pronação das mãos e calcanhar-joelho, quando o paciente apresentou diminuição dos movimentos dos membros direitos. No exame do equilíbrio e da marcha apresentou sinal de Romberg negativo e a marcha do tipo hemiplégica.

No miniexame do estado mental obteve a pontuação de 10 , em um ponto de corte de 26 , da seguinte maneira: Orientação temporal $=0$; Orientação espacial $=0$; Registro (nomeação de objetos) $=1$; Cálculo (subtrair) $=0$; Evocação (três palavras ditas anteriormente $)=2$; Linguagem 1 (nomear um relógio e uma caneta $)=1$; Linguagem 2 (repetir: nem aqui, nem ali, nem lá) $=1$; Linguagem 3 (seguir a um comando) $=3$; Linguagem 4 (ler e obedecer $)=1$; Linguagem 5 (escrever uma frase completa $)=0$; Linguagem 6 (copiar um desenho) $=1$.

\section{Discussão do Caso}

A avaliação e a abordagem inicial da criança na Sala de Emergência são constituídas por procedimentos simples, que, se feitos de forma rápida e ordenada, trarão grande benefício ao paciente. A sequência de atendimento prioriza as lesões que levariam a óbito, mais rapidamente e, por isso, devem ser prioritárias. A conduta realizada no CTI foi de acordo com as diretrizes vigentes de um TCE considerado grave (Glasgow 7), com a abordagem das vias aéreas o paciente foi prontamente entubado, recebeu ventilação mecânica visando a normocapnia, controle de sangramentos externos, exame neurológico (ECG), exposição e avaliação de todo o corpo.

Toda criança com pontuação na ECG, menor ou igual a oito, deve-se assegurar via aérea definitiva, a fim de se evitar a hipóxia, a hipercarpnia e a aspiração. A frequência de complicações nos procedimentos para obtenção da via aérea justifica a utilização de protocolos com medicações para proteção encefálica, anestesia, analgesia e bloqueio neuromuscular. A sequência rápida de intubação é recomendada para evitar aumento da pressão intracraniana (PIC) que ocorre durante a laringoscopia e pela passagem do tubo pela glote. Após a intubação deve-se manter a PCO2 de 35 a $40 \mathrm{mmHg}$, e no paciente em estudo, a PCO2 era de $31,6 \mathrm{mmhg}$, o que explica o procedimento e a hiperventilação, através de efeitos vasoconstritores, que reduzem a pressão intracraniana por períodos específicos. A hipotensão deve ser identificada e corrigida imediatamente, por meio de reposição volêmica, o paciente recebeu hidratação vigorosa. O uso do manitol é uma medida especifica para redução de pressão intracraniana, e deve ser feita no atendimento inicial dos pacientes que apresentam sinais de hipertensão intracraniana (HIC), que são: Tríade de Cushing (Hipertensão arterial, bradicardia e alterações respiratórias), dilatação pupilar unilateral ou pupilas fixas e dilatadas bilateralmente, plegia ou postura motora de decorticação ou descerebração, diminuição de três ou mais pontos na ECG. O seu uso justificou-se, pois o paciente apresentava anisocoria (E>D), e diminuição na ECG.

Rev. de Saúde, Vassouras, v. 1, n. 1, p. 07-14, jan./mar., 2010 
Trauma Cranioencefálico na Infância

Bartira de Godoy Maranhão Santos - Flávia Cristina Malheiros Hayashi - Adriana Vaz de Oliveira e Silva - Cinthia Ferreira da Silva - Rodrigo Eustáquio

Reabilitação e Neurologia

A fenitoína foi usada para prevenir atividades convulsivas que aumentariam o fluxo sangüíneo cerebral e, consequentemente, a pressão intracraniana.

Essas medidas básicas evitam as lesões cerebrais secundárias, que são hipertensão intracraniana, hipotensão e hipóxia, e, a prevenção e tratamento dessas lesões, atualmente, são os principais enfoques do tratamento intensivo moderno a pacientes com TCE, pois estas, quando acontecem, agravam o quadro do paciente, e diferente das lesões primárias, os processos bioquímicos e sistêmicos que constituem as lesões secundárias podem ser amenizadas por uma variedade de intervenções ou terapias farmacológicas.

A tomografia computadorizada de crânio e encéfalo foi realizada rapidamente, assim como exige o protocolo. Foram realizados outros exames de excelência, como a gasometria arterial e o hemograma, usados para evitar e monitorar possíveis lesões secundárias.

Neste caso, foi feito o tratamento conservador no paciente, pois a indicação cirúrgica é controversa, e essa conduta conservadora é uma opção inicial, mesmo quando a lesão se localiza em áreas eloquentes que poderão trazer morbidades, porém, a indicação cirúrgica é necessária quando apresenta aumento significativo de volume e controles radiológicos ou ocorre a piora clínica. O uso de narcóticos e benzodiazepínicos (midazolam) serviram para sedação e paralisia neuromuscular no paciente intubado, pois estímulos nocivos elevam a pressão intra-craniana. A antibioticoterapia profilática (ceftriaxone e oxacilina) foi feita devido à intubação e a permanência no CTI, a fim de evitar pneumonia adquirida em ambiente hospitalar. No entanto, o paciente adquiriu pneumonia grave por acinetobacter, para o que foi feito o uso de imipenem, tetraciclina e meropenem, cuja resolução se deu em 15 dias de tratamento.

Em relação à permanência no hospital, percebeu-se que as medidas terapêuticas se encaixam padrões indicados ao tratamento. No entanto, devido à gravidade de sua contusão temporo-parietal, com hemorragia subaracnóidea e perda de massa encefálica, as sequelas neurológicas e motoras são de grande importância, como a hemiplegia, hipotrofia e hipertonia direita (relacionando ao lobo frontal - responsável pela função motora); a afasia de Broca (relacionados à linguagem - lobo temporal e frontal), desorientação alopísquica (lobo parietal) e amnésia pós-traumática (lobo temporal memória), a assistência ao paciente foi maior.

Pode-se dizer diante do quadro do paciente, que o trauma ocasionou uma lesão piramidal (trato corticoespinhal, no córtex frontal pré-motor, no giro pós-central onde situa-se a área sensitiva e no córtex parietal). Quando há lesão extensa do trato piramidal ocorre hemiplegia contralateral.

$\mathrm{Na}$ síndrome piramidal os reflexos tornam-se exaltados porque os motoneurônios tornam-se hiperativos. A lesão desses tratos ocasiona os denominados sinais de liberação piramidal, entre os quais a hiperreflexia é o mais marcante, e esta é bem significativa no paciente em questão. $\mathrm{O}$ sinal de Babinski encontrado no paciente indica lesão do trato corticospinal, pois este em condições normais inibe a resposta em flexão dorsal.

Rev. de Saúde, Vassouras, v. 1, n. 1, p. 07-14, jan./mar., 2010 
Trauma Cranioencefálico na Infância

Bartira de Godoy Maranhão Santos - Flávia Cristina Malheiros Hayashi - Adriana Vaz de Oliveira e Silva - Cinthia Ferreira da Silva - Rodrigo Eustáquio

Reabilitação e Neurologia

As alterações oculares do globo ocular do paciente, acontecem pois, a inervação da musculatura extrínseca ocular, que é responsável por movimentos como adução, abdução, elevação e abaixamento, depende dos nervos oculomotor (III), troclear (IV) e abducente (VI). O núcleo do III nervo localiza-se numa situação muito vulnerável a compressões pela porção medial do lobo temporal. A lesão dos neurônios do nervo facial, quer do corpo celular localizado no núcleo, quer dos axônios em qualquer parte do trajeto, causa paralisia facial periférica, o que causou a redução da mímica facial do paciente.

Afasia global resulta de lesão que envolve a área de Broca. L.T.M apresentou expressão e a compreensão verbal reduzidas a poucas palavras,afasia nominal ou amnéstica.

Atualmente, de acordo com relatos da mãe, o paciente encontra-se sob acompanhamento da fonaudiologia, fisioterapia e neurologista, havendo melhora considerável nos sistemas comprometidos e consequentemente das sequelas.

\section{Conclusão}

A medicina preventiva é de importância única e insubstituível. O traumatismo crânio encefálico, por ser a primeira causa de morbidade e mortalidade mundial, merece atenção especial nesse aspecto. Grande parte destes fatores é corrigível ou evitável, com reanimação hídrica inicial agressiva, tratamento cirúrgico em tempo hábil, monitoração e cuidados intensivos adequados, além da disseminação de informações que contribuam para o planejamento de ações preventivas que poderiam reduzir sensivelmente o número de casos de TCE em crianças e adolescentes. Muitas vezes, as crianças com TCE sofrem em silêncio, sem consciência das profundas modificações que ocorreram no seu pensamento e comportamento. O TCE pode, frequentemente, ser invisível a olho nu, o que não o torna ausente. Pode ser subdiagnosticado, embora poderoso, resulte em grandes e profundas consequências para crianças e adolescentes. $\mathrm{O}$ seu poder não deve nunca ser subestimado. Apesar de grande número de trabalhos publicados, os fatores relacionados com um melhor prognóstico em crianças ainda permanecem obscuros. 
Trauma Cranioencefálico na Infância

Bartira de Godoy Maranhão Santos - Flávia Cristina Malheiros Hayashi - Adriana Vaz de Oliveira

e Silva - Cinthia Ferreira da Silva - Rodrigo Eustáquio

Reabilitação e Neurologia

\section{Referências}

Nitrini, Ricardo; Bacheschi, Luiz Alberto. A neurologia que todo médico deve saber. São Paulo: Atheneu, 2005.

Patten, John. Diagnóstico diferencial em neurologia. São Paulo: Roca, 1985.

Freire, Evandro. Trauma: a doença dos séculos. São Paulo: Atheneu, 2001.

Bongard, Fred S. (Org.). Terapia intensiva: diagnóstico e tratamento. 2. ed. São Paulo: Artmed, 2006.

Paiva, Wellingson et al. Contusões cerebrais devido a trauma craniencefálico: princípios fisiopatológicos e conduta. Jornal Brasileiro de Medicina, Rio de Janeiro, v. 91, n. 2, p. 11-19, ago. 2006.

Carvalho LF; Affonseca C; Guerra S; Ferreira A; Goulart E. Traumatismo cranioencefálico grave em crianças e adolescentes. Rev. bras. ter. intensiva vol.19 no.1 São Paulo Jan./Mar. 2007

Melo JR, Filho JO, Silva R, Moreira Júnior ED. Fatores Preditivos do Prognóstico em vítimas de truama cranioencefálico. Arq. Neuropsiquiatria 2005;63(4):1054-1057

Tavares MP. Tratamento Clínico do Traumatismo Crânio-Encefálico. Neurosurgery

Macedo KC, Características Clínicas e Epidemiológicas de crianças e adolescentes com Traumatismo Cranioencefálico leve e Análise de fatores associados à fratura de crânio e lesão intracraniana. Dissertação de mestrado, Faculdade de Medicina Universidade federal de Minas Gerais, Belo Horizonte,2006.

Lobato de Faria Mt.. Abordagem multidisciplinar no acompanhamento de uma criança com Traumatismo Crânio-Encefálico Análise Psicológica (2006), 2 (XXIV): 235245.

Batista SE; Baccani JG ; Silva RA; Gualda K; Vianna RJ; Análise comparativa entre os mecanismos de trauma, As lesões e o perfil de gravidade das vítimas, em Catanduva-SP Rev. Col. Bras. Cir. Vol. 33 - No 1, Jan. / Fev. 2006.'

Rev. de Saúde, Vassouras, v. 1, n. 1, p. 07-14, jan./mar., 2010 